\title{
ON THE NILPOTENT RANKS OF CERTAIN SEMIGROUPS OF TRANSFORMATIONS
}

\author{
by G. U. GARBA
}

(Received 5 November, 1991)

1. Introduction. Let $P_{n}$ be the semigroup of all partial transformations on the set $X_{n}=\{1, \ldots, n\}$. As usual, we shall say that an element $\alpha$ in $P_{n}$ is of type $(k, r)$ or belongs to the set $[k, r]$ if $|\operatorname{dom} \alpha|=k$ and $|\operatorname{im} \alpha|=r$. The completion $\alpha^{*}$ of an element $\alpha \in[n-1, n-1]$ is an element in $[n, n]$ defined by

$$
i \alpha^{*}=j, \quad x \alpha^{*}=x \alpha \text { otherwise, }
$$

where $\{i\}=X_{n} \backslash$ dom $\alpha$ and $\{j\}=X_{n} \backslash \operatorname{im} \alpha$.

For $n$ even, the subsemigroup $\mathrm{SI}_{n}$ of $P_{n}$ consisting of all strictly partial one-one transformations was proved to be nilpotent-generated by Gomes and Howie [2]. If $n$ is odd, they showed that the nilpotents in $\mathrm{SI}_{n}$ generate $\mathrm{SL}_{n} \backslash W_{n-1}$, where $W_{n-1}$ consists of all $\alpha \in[n-1, n-1]$ whose completions are odd permutations.

Simultaneously and independently, Sullivan [7] showed that the subsemigroup $\mathrm{SP}_{n}$ of $P_{n}$ consisting of all strictly partial transformations of $X_{n}$ is nilpotent-generated if $n$ is even. If $n$ is odd, the nilpotents in $\mathrm{SP}_{n}$ generate $\mathrm{SP}_{n} \backslash W_{n-1}$.

The rank of a semigroup $S$ is the cardinality of any subset $A$ of minimal order in $S$ such that $\langle A\rangle=S$. If the generating set $A$ consists of nilpotent elements only, then we shall refer to the cardinality of $A$ as the nilpotent rank of $S$. Since one of the semigroups we will be considering is an inverse semigroup, we would like to clarify the notion of a generating set in an inverse semigroup. Given a subset $A$ in an inverse semigroup $S$, we shall always want to consider the smallest inverse subsemigroup containing $A$. In effect this is the set of all finite products of elements of $A$ and their inverses. Following [3], we shall use the notation $\langle\langle A\rangle\rangle$ for this inverse subsemigroup. Accordingly, by the rank of an inverse semigroup $S$ we shall mean the cardinality of any subset $A$ of minimal order in $S$ such that $\langle\langle A\rangle\rangle=S$.

Let $N$ and $M$ be the sets of all nilpotent elements in $\mathrm{SI}_{n}$ and $\mathrm{SP}_{n}$ respectively. In [3], Gomes and Howie proved that the rank and the nilpotent rank of $\langle\langle N\rangle\rangle$ are both equal to $n+1$ for all $n$, and in [1], Garba showed that the rank and the nilpotent rank of $\langle M\rangle$ are both equal to $n+2$ for all $n$. In Section 2 we generalize the results of Gomes and Howie [3] (in line with Howie and McFadden [6]) by showing that if $1 \leq r \leq n-2$ then the rank and the nilpotent rank of the inverse semigroup

$$
U(n, r)=\left\{\alpha \in \mathrm{SI}_{n}:|\operatorname{im} \alpha| \leq r\right\}
$$

are both equal to $\left(\begin{array}{l}n \\ r\end{array}\right)+1$. In Section 3 we generalize the results of Garba [1] by showing that if $1 \leq r \leq n-2$ then the rank and the nilpotent rank of the semigroup

$$
V(n, r)=\left\{\alpha \in \mathrm{SP}_{n}:|\operatorname{im} \alpha| \leq r\right\}
$$

are both equal to $(r+1) S(n, r+1)$, where $S(n, r+1)$ is the Stirling number of the second kind, defined by

$$
S(n, 1)=S(n, n)=1, \quad S(n, r)=S(n-1, r-1)+r S(n-1, r) .
$$

For standard terms in semigroup theory see [4]. In all that follows, we consider $n \geq 3$.

Glasgow Math. J. 36 (1994) 1-9. 


\section{One-one partial transformations.}

Lемма 2.1. For all $r \leq n-2$, we have

$$
J_{r} \subseteq\left(N \cap J_{r}\right)^{2}
$$

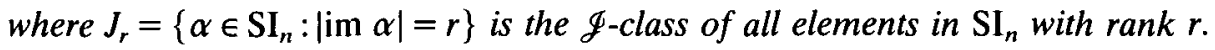

Proof. The result is trivial for $r \leq 1$. If $r \geq 2$ then the result follows from Remark 3.16 in [2], where the authors prove that $J_{n-2} \subseteq\left(N \cap J_{n-2}\right)^{2}$, and from Lemma 4.1 in the same paper, which states that if $J_{r} \subseteq\left(N \cap J_{r}\right)^{k}$ then $J_{r-1} \subseteq\left(N \cap J_{r-1}\right)^{k}$ for $2 \leq r \leq n-1$.

It follows from this lemma that the nilpotents in $J_{r}$ generate $U(n, r)$.

Denote by $P_{r}$ the principal factor $U(n, r) / U(n, r-1)$. Then $P_{r}$ may be thought of in the usual way as $J_{r} \cup\{0\}$. Also, $P_{r}$ has $\left(\begin{array}{c}n \\ r\end{array}\right)$ non-null $\mathscr{R}$-classes corresponding to the $\left(\begin{array}{c}n \\ r\end{array}\right)$ possible domains of cardinality $r$, and $\left(\begin{array}{c}n \\ r\end{array}\right)$ non-null $\mathscr{L}$-classes corresponding to the $\left(\begin{array}{c}n \\ r\end{array}\right)$ possible images. It is a Brandt semigroup isomorphic to $B\left(S_{r},\{1, \ldots, m\}\right)$, where $S_{r}$ is the symmetric group on $X_{r}$ and $m=\left(\begin{array}{c}n \\ r\end{array}\right)$. Hence, since the rank of $S_{r}$ is known to be 2 , it follows by Theorem 3.3 in [3] that $P_{r}$ has inverse semigroup rank $\left(\begin{array}{c}n \\ r\end{array}\right)+1$.

From [2], we borrow the notation $\left\|a_{1} a_{2} \ldots a_{r+1}\right\|(1 \leq r \leq n-1)$ for the nilpotent $\alpha$ with domain $\left\{a_{1}, \ldots, a_{r}\right\}$ and image $\left\{a_{2}, \ldots, a_{r+1}\right\}$ for which $a_{i} \alpha=a_{i+1}(i=1, \ldots, r)$. We shall refer to these type of nilpotents as primitive in the next section.

Theorem 2.2. Let $n \geq 3$ and let $r \leq n-2$. Then

$$
\operatorname{rank}\left\langle\langle U(n, r\rangle\rangle=\operatorname{nilrank}\langle\langle U(n, r)\rangle\rangle= \begin{cases}\left(\begin{array}{l}
n \\
r
\end{array}\right)+1 & \text { if } r \geq 3, \\
\left(\begin{array}{l}
n \\
r
\end{array}\right) & \text { if } r=2, \\
n-1 & \text { if } r=1 .\end{cases}\right.
$$

Proof. From the fact that $P_{r}$ (as an inverse semigroup) has rank $\left(\begin{array}{c}n \\ r\end{array}\right)+1$ it follows that rank $\langle\langle U(n, r)\rangle\rangle \geq\left(\begin{array}{c}n \\ r\end{array}\right)+1$. To complete the proof we must find a generating set of $\langle\langle U(n, r)\rangle\rangle$ consisting of $\left(\begin{array}{l}n \\ r\end{array}\right)+1$ nilpotents.

Let $A_{1}, A_{2}, \ldots, A_{m}$ be a list of the subsets of $X_{n}$ of cardinality $r$. Thus $m=\left(\begin{array}{c}n \\ r\end{array}\right)$. Let $H_{A_{i}, A_{j}}$ denote the $\mathscr{H}$-class in $J_{r}$ consisting of all the elements whose domain is $A_{i}$ and image $A_{j}(i, j=1,2, \ldots, m)$. Suppose that $A_{1}=\{1,2, \ldots, r\}$. Then the $\mathscr{H}$-class $H_{A_{1}, A_{1}}$ is the symmetric group on $\{1,2, \ldots, r\}$, and if $r \geq 3$ then it is generated by the elements $\sigma, \tau$, where

$$
\sigma=(12), \quad \tau=(12 \ldots r) \text {. }
$$

We now show that each of $\sigma, \tau$ can be expressed as a product of nilpotents. For this purpose, we will suppose that $A_{2}=\{2, \ldots, r, r+1\}, A_{3}=\{1, \ldots, r-1, r+1\}$ and $A_{4}=\{2, \ldots, r-1, r+1, r+2\}$. The proof depends on whether $r$ is odd or even. For $r$ odd we have

$$
\sigma=\alpha_{2}^{-1} \beta \alpha_{3} \quad \text { and } \quad \tau=\gamma_{2}^{-1} \alpha_{2}
$$

where

$$
\begin{aligned}
\alpha_{2} & =\|r+1 r r-1 \ldots 21\| \in H_{A_{2}, A_{1}}, \\
\beta & =\|r r-2 r-4 \ldots 3 r+1 r-1 \ldots 421\| \in \dot{H}_{A_{2}, A_{3}}, \\
\alpha_{3} & =\|r+112 \ldots r\| \in H_{A_{3}, A_{1}}, \\
\gamma_{2} & =\|r+1 r-1 \ldots 2 r r-2 \ldots 31\| \in H_{A_{2}, A_{1}} .
\end{aligned}
$$


If for this case we now choose a nilpotent $\alpha_{i} \in H_{A_{i}, A_{1}}$ for $i=4, \ldots, m$ in an arbitrary way, we see that

$$
\sigma, \tau, \alpha_{2}, \ldots, \alpha_{m} \in\left\langle\left\langle\alpha_{2}, \ldots, \alpha_{m}, \beta, \gamma_{2}\right\rangle\right\rangle .
$$

By the remark before Theorem 3.3 in [3], the elements $\sigma, \tau, \alpha_{2}, \ldots, \alpha_{m}$ generate $P_{r}$. It follows that $P_{r}$, and hence also $U(n, r)$ is generated by the $m+1$ nilpotents $\alpha_{2}, \ldots, \alpha_{m}$, $\beta, \gamma_{2}$ provided $r$ is odd.

For $r$ even we have

where

$$
\sigma=\alpha_{3}^{-1} \beta \alpha_{4} \quad \text { and } \quad \tau=\gamma_{4}^{-1} \alpha_{4},
$$

$$
\begin{aligned}
\alpha_{3} & =\|r+123 \ldots r-2 r-11 r\| \in H_{A_{3}, A_{1}}, \\
\beta & =\|1 r-2 r+13254 \ldots r-5 r-6 r-3 r-4 r-1 r+2\| \in H_{A_{3}, A_{4}}, \\
\alpha_{4} & =\|r+224 \ldots r\| \cup\|r+1 r-1 \ldots 31\| \in H_{A_{4}, A_{1},} \\
\gamma_{4} & =\|r+1 r-2 r-1 r-4 r-3 r-6 r-5 \ldots 9674523 r\| \cup\|r+21\| \in H_{A_{4}, A_{1}} .
\end{aligned}
$$

In this case $P_{r}$ and hence $U(n, r)$ is generated by the $m+1$ nilpotents $\alpha_{2}, \ldots, \alpha_{m}, \beta, \gamma_{4}$, where $\alpha_{i} \in H_{A_{i}, A_{1}}$ are chosen arbitrarily for $i=2,5,6, \ldots, m$.

It now remains to show that the result is true for $r=2$ and $r=1$.

If $r=2, S_{2}$ is cyclic and thus has only one generator. For this case we will suppose that $A_{1}=\{1,2\}$ and $A_{m}=\{n-1, n\}$. The $\mathscr{C}$-class $H_{A_{1}, A_{1}}$ is the symmetric group on $A_{1}$ and is generated by

$$
\sigma=(12)
$$

Now,

$$
\sigma=\gamma_{m}^{-1} \alpha_{m}
$$

where

$$
\begin{aligned}
& \alpha_{m}=\|n-12\| \cup\|n 1\| \in H_{A_{m}, A_{1}}, \\
& \gamma_{m}=\|n-11\| \cup\|n 2\| \in H_{A_{m}, A_{1}} .
\end{aligned}
$$

So, if we choose nilpotents $\alpha_{2}, \ldots, \alpha_{m-1}$ as in the above cases, we see that $\alpha_{2}, \ldots, \alpha_{m}$, $\gamma_{m}$ generate $U(n, r)$. Thus $U(n, r)$ has rank $1+m-1=m$.

If $r=1$, the symmetric group $S_{1}$ has rank 0 , and it is easy to verify that the following $n-1$ nilpotents generate $U(n, r)$ :

$$
\|21\|,\|31\|,\|41\|, \ldots,\|n 1\| .
$$

3. Partial transformations. The semigroup $V(n, r)$ has $r+1 \mathscr{g}$-classes, namely $J_{r}$, $J_{r+1}, \ldots, J_{0}$ (where $J_{0}$ consists of the empty map). For each $t$ such that $1 \leq t \leq r$ we have

$$
J_{t}=\bigcup_{k=t}^{n-1}[k, t] .
$$

The number of $\mathscr{L}$-classes in the $\mathscr{E}$-class $J_{r}$ of $V(n, r)$ is the number of image sets in $X_{n}$ of cardinality $r$, namely $\left({ }_{r}^{n}\right)$, and the number of $\mathscr{R}$-classes in $J_{r}$ is the number of equivalence relations $\rho$ on each of the subsets $A$ of cardinality $k$ (where $n-1 \geq k \geq r$ ) for 
which $|A / \rho|=r$, and this number is

$$
\begin{aligned}
\sum_{k=r}^{n-1}\left(\begin{array}{l}
n \\
k
\end{array}\right) S(k, r) & =\sum_{k=r}^{n}\left(\begin{array}{l}
n \\
k
\end{array}\right) S(k, r)-S(n, r) \\
& =S(n+1, r+1)-S(n, r) \\
& =(r+1) S(n, r+1) .
\end{aligned}
$$

Like $U(n, r)$, the semigroup $V(n, r)$ is generated by the nilpotent elements in $J_{r}$ (see Lemma 2.3 in [1]). We also have from Lemma 3 in [6] that for $2 \leq r \leq n-2$,

$$
\operatorname{rank}(V(n, r)) \geq(r+1) S(n, r+1) \text {. }
$$

TheOREM 3.1. For $n \geq 3$ and $2 \leq r \leq n-2$, we have

$$
\operatorname{rank}(V(n, r))=\operatorname{nilrank}(V(n, r))=(r+1) S(n, r+1) \text {. }
$$

The proof depends on the following lemma.

Lemma 3.2. Suppose that we can arrange the subsets $A_{1}, \ldots, A_{m}$ (where $m=\left(\begin{array}{c}n \\ r\end{array}\right)$ and $2 \leq r \leq n-2)$ of $X_{n}$ of cardinality $r$ in such a way that $\left|A_{i} \cap A_{i-1}\right|=r-1$ for $i=$ $1, \ldots, m-1$ and $\left|A_{m} \cap A_{1}\right|=r-1$. Then there exist nilpotents $\alpha_{1}, \ldots, \alpha_{p}$ (where $p=(r+1) S(n, r+1))$ such that $\left\{\alpha_{1}, \ldots, \alpha_{p}\right\}$ is a set of generators for $V(n, r)$.

Proof. Notice first that every element $\alpha \in[k, r], r<k \leq n-1$, is expressible as a product of a nilpotent in its own $\mathscr{R}$-class and an element in $[r, r]$. For

$$
\alpha=\left(\begin{array}{ccccc}
A_{1} & A_{2} & \ldots & A_{r-1} & A_{r} \\
a_{2} & a_{3} & \ldots & a_{r} & x
\end{array}\right)\left(\begin{array}{ccccc}
a_{2} & a_{3} & \ldots & a_{r} & x \\
b_{1} & b_{2} & \ldots & b_{r-1} & b_{r}
\end{array}\right)
$$

where

$$
\alpha=\left(\begin{array}{ccc}
A_{1} & \ldots & A_{r} \\
b_{1} & \ldots & b_{r}
\end{array}\right)
$$

$a_{i} \in A_{i}$ for all $i \in\{2, \ldots, r\}$ and $x \in X_{n} \backslash$ dom $\alpha$.

In the arrangement of our subsets $A_{1}, \ldots, A_{m}$ we shall assume that $A_{1}=\{n-r+1$, $n-r+2, \ldots, n\}, A_{2}=\{n-r, \ldots, n-1\}$ and $A_{m}=\{1, n-r+2, \ldots, n\}$. We shall also represent any two adjacent subsets $A_{i}, A_{i+1}$ by the two subsets $\left\{x_{1}, \ldots, x_{r-1}, y_{i}\right\}$ and $\left\{x_{1}, \ldots, x_{r-1}, z_{i+1}\right\}$, where $z_{i+1} \neq y_{i}$, and $z_{i+1}, y_{i} \neq x_{i}$ for any $i$. Define $H_{A_{i}, A_{j}}$ to consist of all elements $\alpha \in[r, r]$ for which $\operatorname{dom} \alpha=A_{i}$ and $\operatorname{im} \alpha=A_{j}$. For $i=1, \ldots, m$ define a mapping $\xi_{i} \in H_{A_{i}, A_{m}}$ as follows:

$$
\begin{gathered}
\xi_{1}=\left(\begin{array}{crrcc}
n-r+1 & n-r+2 & \ldots & n \\
1 & n-r+2 & \ldots & n
\end{array}\right), \\
\xi_{2}=\left(\begin{array}{ccccc}
n-r & n-r+1 & n-r+2 & \ldots & n-1 \\
n-r+2 & 1 & n-r+3 & \ldots & n
\end{array}\right),
\end{gathered}
$$

and for $i=2, \ldots, m-1$ if

$$
\xi_{i}=\left(\begin{array}{ccccc}
x_{1} & x_{2} & \ldots & x_{r-1} & y_{i} \\
t_{1} & t_{2} & \ldots & t_{r-1} & t_{r}
\end{array}\right)
$$


define

$$
\xi_{i+1}=\left(\begin{array}{ccccc}
x_{1} & x_{2} & \ldots & x_{r-1} & z \\
t_{2} & t_{3} & \ldots & t_{r} & t_{1}
\end{array}\right)
$$

Then it is easy to see that the mapping

$$
\phi: B\left(S_{r},\{1, \ldots, m\}\right) \rightarrow Q_{r}
$$

defined by $(i, \eta, j) \phi=\xi_{i} \eta \xi_{j}^{-1}$ is an isomorphism. Here $S_{r}$ is the symmetric group on $\{1, n-r+2, \ldots, n\}, Q_{r}$ is the principal factor

$$
[r, r] / \bigcup_{l=0}^{r-1}[l, l] \simeq[r, r] \cup\{0\} .
$$

From Proposition 2.4 in [1], the set

$$
T=\left\{\left(1, g_{1}, 1\right),\left(1, g_{2}, 2\right),(2, e, 3), \ldots,(m-1, e, m),(m, e, 1)\right\},
$$

where $g_{1}=(1 n-r+2 \ldots n), g_{2}=(1 n-r+2)$ and $e$ is the identity permutation in $S_{r}$, generates $B\left(S_{r},\{1, \ldots, m\}\right)$. Thus $T \phi$ generates $Q_{r}$ and hence $[r, r]$. If we now define

$$
\alpha_{1}=\xi_{1} g_{2} \xi_{2}^{-1}, \alpha_{i}=\xi_{i} \xi_{i+1}^{-1} \quad \text { for } i=2, \ldots, m-1
$$

and

$$
\beta=\xi_{m} \xi_{1}^{-1}, \quad \delta=\xi_{1} g_{1} \xi_{1}^{-1},
$$

we obtain a generating set $\left\{\beta, \delta, \alpha_{1}, \ldots, \alpha_{m-1}\right\}$ of $[r, r]$, where

$$
\begin{gathered}
\alpha_{1}=\|n n-1 \ldots n-r+1 n-r\|, \\
\alpha_{i}=\left\|y_{i} x_{r-1} \ldots x_{1} z_{i+1}\right\| \quad \text { for } i=2, \ldots, m-1
\end{gathered}
$$

are all nilpotents. On the other hand,

$$
\delta=\left(\begin{array}{ccccc}
n-r+1 & n-r+2 & \ldots & n-1 & n \\
n-r+2 & n-r+3 & \ldots & n & n-r+1
\end{array}\right)
$$

is clearly non-nilpotent. However if $r$ is odd we have

$$
\delta=\alpha_{1} \lambda_{1}
$$

where

$$
\lambda_{1}=\|n-r n-r+2 \cdots n-1 n-r+1 n-r+3 \cdots n-2 n\| .
$$

If $r$ is even, and is of the form $4 q+2(q \geq 0)$, then

$$
\delta=\alpha_{1} \eta_{1} \eta_{2}
$$

where

$$
\begin{aligned}
\eta_{1}= & \|n-r+1 n-r+5 \ldots n-1 n-r+3 n-r+7 \ldots n-3 n-r n-r+4 \ldots n-21\| \\
& \bigcup\|n-r+2 n-r+6 \ldots n\|
\end{aligned}
$$

and

$$
\eta_{2}=\|n-r n-1 n-3 \ldots n-r+3 n-r+1\| \cup\|1 n n-2 n-4 \ldots n-r+2\| .
$$

If $r$ is even and of the form $4 q(q \geq 1)$ then

$$
\delta=\alpha_{1} \psi_{1} \psi_{2}
$$


where

$$
\psi_{1}=\|n-r+1 n-r n-r+3 n-r+2 n-r+5 n-r+4 \ldots n-1 n-21\|
$$

and

$$
\psi_{2}=\|n-r n-r+3 n-r+2 n-r+5 \ldots n-2 n-r+1\| \cup\|1 n\| .
$$

Next, $\beta$ may or may not be nilpotent. However, as $\beta \in[r, r]$, if $\beta$ is non-nilpotent, then by Lemma 2.1 it is expressible as a product of two nilpotents in $[r, r]$, say

$$
\beta=\zeta_{1} \zeta_{2} \text {. }
$$

It is clear that $\beta \mathscr{R} \zeta_{1}$ and $\beta \mathscr{L} \zeta_{2}$, that is $R_{\zeta_{1}}=A_{m}$ and $L_{\zeta_{2}}=A_{1}$.

We now define $\lambda_{1}^{\prime}, \eta_{1}^{\prime}, \eta_{2}^{\prime}, \psi_{1}^{\prime}, \psi_{2}^{\prime}$ and $\zeta_{2}^{\prime}$ as follows:

$$
\begin{gathered}
\lambda_{1}^{\prime}=\lambda_{1} \cup(1, n), \\
\eta_{1}^{\prime}=\eta_{1} \cup(n, 1), \quad \eta_{2}^{\prime}=\eta_{2} \cup(n-r+1, n), \\
\psi_{1}^{\prime}=\psi_{1} \cup(n, n-2), \quad \psi_{2}^{\prime}=\psi_{2} \cup(n, n-r+1) .
\end{gathered}
$$

Before we define $\zeta_{2}^{\prime}$, we note that from Theorem 2.8 in [2], $\zeta_{2}$ can be expressed as a disjoint union of $k$ primitive nilpotents, say

$$
\zeta_{2}=\mu_{1} \cup \mu_{2} \cup \ldots \cup \mu_{k} \text {. }
$$

If $k \geq 2$, then assume

$$
\mu_{1}=\left\|x_{1} \ldots x_{s}\right\| \quad \text { and } \quad \mu_{2}=\left\|y_{1} \ldots y_{t}\right\|
$$

and define $\zeta_{2}^{\prime}$ as

$$
x \zeta_{2}^{\prime}=x \zeta_{2} \quad \text { if } \quad x \in \operatorname{dom} \zeta_{2}
$$

and

$$
x_{s} \zeta_{2}^{\prime}=y_{t}
$$

On the other hand if $k=1$ then $\left|\operatorname{dom} \zeta_{2} \cup \operatorname{im} \zeta_{2}\right|=r+1$, and since $r \leq n-2$ we have $X_{n} \backslash\left(\operatorname{dom} \zeta_{2} \cup \operatorname{im} \zeta_{2}\right)$ to be non-empty. Then define $\zeta_{2}^{\prime}$ as

$$
\zeta_{2}^{\prime}=\zeta_{2} \cup(x, n-r+1)
$$

where $x \in X_{n} \backslash\left(\operatorname{dom} \zeta_{2} \cup \operatorname{im} \zeta_{2}\right)$.

Note that $\lambda_{1}^{\prime}, \eta_{1}^{\prime}, \eta_{2}^{\prime}, \psi_{1}^{\prime}, \psi_{2}^{\prime}$ and $\zeta_{2}^{\prime}$ are distinct, and belong to $[r+1, r]$. If we now replace $\lambda_{1}, \eta_{1}, \eta_{2}, \psi_{1}$ and $\psi_{2}$ by $\lambda_{1}^{\prime}, \eta_{1}^{\prime}, \eta_{2}^{\prime}, \psi_{1}^{\prime}$ and $\psi_{2}^{\prime}$ respectively in equations (3.3)-(3.5) then it is easy to see that the equations remain unaltered. Since $\beta, \zeta_{1}, \zeta_{2}$ are all one-one and of the same height, we must have

$$
\operatorname{dom} \beta=\operatorname{dom} \zeta_{1}, \quad \operatorname{im} \zeta_{1}=\operatorname{dom} \zeta_{2},
$$

and since $x_{s}, x \notin \operatorname{dom} \zeta_{2}=\operatorname{im} \zeta_{1}$ we conclude that

$$
\zeta_{1} \zeta_{2}=\zeta_{1} \zeta_{2}^{\prime}
$$

Now, if $\beta$ is nilpotent then $V(n, r)$ is generated by

$$
\left\{\beta, \lambda_{1}^{\prime}, \alpha_{1}, \ldots, \alpha_{p-2}\right\}, \quad\left\{\beta, \eta_{1}^{\prime}, \eta_{2}^{\prime}, \alpha_{1}, \ldots, \alpha_{p-3}\right\}
$$


or

$$
\left\{\beta, \psi_{1}^{\prime}, \psi_{2}^{\prime}, \alpha_{1}, \ldots, \alpha_{p-3}\right\}
$$

according to whether $r$ is odd, even and of the form $4 q+2(q \geq 0)$ or even and of the form $4 q(q \geq 1)$, and $\alpha_{m}, \ldots, \alpha_{p-k}(k=2,3)$ are chosen arbitrarily to cover all the $\mathscr{R}$-classes in $J_{r}$.

If $\beta$ is non-nilpotent, then $V(n, r)$ is generated by

$$
\left\{\zeta_{1}, \zeta_{2}^{\prime}, \lambda_{1}^{\prime}, \alpha_{1}, \ldots, \alpha_{p-3}\right\}, \quad\left\{\zeta_{1}, \zeta_{2}^{\prime}, \eta_{1}^{\prime}, \eta_{2}^{\prime}, \alpha_{1}, \ldots, \alpha_{p-4}\right\}
$$

or

$$
\left\{\zeta_{1}, \zeta_{2}^{\prime}, \psi_{1}^{\prime}, \psi_{2}^{\prime}, \alpha_{1}, \ldots, \alpha_{p-4}\right\}
$$

according to whether $r$ is odd, even and of the form $4 q+2(q \geq 0)$ or even and of the form $4 q(q \geq 1)$, and $\alpha_{m}, \ldots, \alpha_{p-k}(k=3,4)$ are chosen arbitrarily to cover all the $\mathscr{R}$-classes in $J_{r}$.

To conclude the proof of Theorem 3.1, it remains to prove that the listing of the subsets of $X_{n}$ of cardinality $r$ as postulated in the statement of Lemma 3.2 can actually be carried out. Let $n \geq 4$ and $2 \leq r \leq n-2$, and consider the following proposition.

$(\mathbf{P}(n, r))$ : there is a way of listing the subsets of $X_{n}$ of cardinality $r$ as $A_{1}, A_{2}, \ldots, A_{m}$ (with $m=\left(\begin{array}{l}n \\ r\end{array}\right), \quad A_{1}=\{n-r+1, \ldots, n\}, A_{2}=\{n-r, \ldots, n-1\}, A_{m}=\{1, n-r+$ $2, \ldots, n\}$ ) such that $\left|A_{i} \cap A_{i+1}\right|=r-1$ for $i=1, \ldots, m-1$ and $\left|A_{m} \cap A_{1}\right|=r-1$.

We shall prove this by a double induction on $n$ and $r$, the key step being a kind of Pascal's Triangle implication.

$$
\mathbf{P}(n-1, r-1) \text { and } \mathbf{P}(n-1, r) \Rightarrow \mathbf{P}(n, r) .
$$

First, however, we anchor the induction with two lemmas.

LEMma 3.7. $\mathbf{P}(n, 2)$ holds for every $n \geq 4$.

Proof. Consider the following arrangement of the subsets of $X_{n}$ of cardinality 2.

$$
\begin{aligned}
& \{1,2\}, \quad\{1,3\}, \ldots, \quad\{1, n-1\}, \quad\{1, n\}, \\
& \{2,3\}, \ldots, \quad\{2, n-1\}, \quad\{2, n\}, \\
& \{n-2, n-1\}, \quad\{n-2, n\}, \\
& \{n-1, n\} \text {. }
\end{aligned}
$$

If we denote the first row by $R_{1}$, second row by $R_{2}$, etc., then we note that the first entry in $R_{i}$ is $\{i, i+1\}$ and the last entry is $\{i, n\}$. Thus the number of elements in $R_{i}$ is $n-i$, and the total number of subsets in all the rows is

$$
\sum_{i=1}^{n-1}(n-i)=\frac{n}{2}(n-1)=\left(\begin{array}{l}
n \\
2
\end{array}\right) .
$$

Hence above is a complete list of the subsets of $X_{n}$ of cardinality 2 .

Note that for any two subsets $A_{s}, A_{r}$ in $R_{i}, A_{s} \cup A_{r}=\{i\}$, and the intersection of the last entry in $R_{i+1}$ with the first entry in $R_{i}$ is $\{i+1\}$. Hence the following arrangement satisfies $\mathbf{P}(n, 2)$ :

$$
R_{n-1}, R_{n-2}, \ldots, R_{i+1}, R_{i}, \ldots, R_{2}, R_{1}
$$


That is, the list begins with all the subsets in $R_{n-1}$, followed by the subsets in $R_{n-2}$, followed by the subsets in $R_{n-3}$, and so on, until $R_{1}$ is reached.

LEMMA 3.8. $\mathbf{P}(n, n-2)$ holds for every $n \geq 4$.

Proof. Note that $\mathbf{P}(4,2)$ follows from Lemma 3.7. So we will assume that $n \geq 5$. Let $R_{i}^{\prime}$ be the list of the complements of the subsets in $R_{i}$ (defined in the proof of Lemma 3.7) arranged in the same order as in $R_{i}$. Let $\left(R_{i}^{\prime}\right)^{-1}$ be $R_{i}^{\prime}$ arranged in the reverse order. For example

$$
\begin{gathered}
R_{n-2}=\{n-2, n-1\},\{n-2, n\}, \\
R_{n-2}^{\prime}=\{1, \ldots, n-3, n\},\{1, \ldots, n-3, n-1\}, \\
\left(R_{n-2}^{\prime}\right)^{-1}=\{1, \ldots, n-3, n-1\},\{1, \ldots, n-3, n\} .
\end{gathered}
$$

Let $T=\{1,3\},\{1,4\}, \ldots,\{1, n-1\}$ and $T^{\prime}=R_{1}^{\prime} \backslash\left(\{1,2\}^{\prime},\{1, n\}^{\prime}\right)$.

It is clear that, for any two subsets $A_{s}^{\prime}, A_{r}^{\prime}$ in $R_{i}^{\prime}$, we have $\left|A_{s}^{\prime} \cap A_{r}^{\prime}\right|=n-3$, and the intersection of the last subset in $R_{i+1}^{\prime}$ and the first subset in $R_{i}^{\prime}$ also contains $n-3$ elements. We also have $n-3$ elements in the intersection of the last subset in $R_{3}^{\prime}$ with the first subset in $\left(R_{2}^{\prime}\right)^{-1}$, and the same number of elements in the intersection of the last subset in $T^{\prime}$ with the subset in $R_{n-1}^{\prime}$. We now have the following arrangement satisfying $\mathbf{P}(n, n-2)$ :

$$
A_{1}^{\prime}, A_{2}^{\prime}, T^{\prime}, R_{n-1}^{\prime}, R_{n-2}^{\prime}, \ldots, R_{3}^{\prime},\left(R_{2}^{\prime}\right)^{-1},
$$

where $A_{1}^{\prime}=\{1,2\}^{\prime}$ and $A_{2}^{\prime}=\{1, n\}^{\prime}$.

Lemma 3.9. Let $n \geq 6$ and $3 \leq r \leq n-3$. Then $\mathbf{P}(n-1, r-1)$ and $\mathbf{P}(n-1, r)$ together imply $\mathbf{P}(n, r)$.

Proof. From the assumption $\mathbf{P}(n-1, r)$ we have a list $A_{1}, \ldots, A_{m}$ (where $m=$ $\left.\left(\begin{array}{c}n-1 \\ r\end{array}\right)\right)$ of the subsets of $X_{n-1}$ with cardinality $r$ such that $\left|A_{1} \cap A_{i+1}\right|=r-1$ for $i=1, \ldots, m-1$, and

$$
A_{1}=\{n-r, \ldots, n-1\}, A_{2}=\{n-r-1, \ldots, n-2\}, A_{m}=\{1, n-r+1, \ldots, n-1\} .
$$

From the assumption $\mathbf{P}(n-1, r-1)$, we have a list $B_{1}, \ldots, B_{t}$ (where $t=\left(\begin{array}{c}n-1 \\ r-1\end{array}\right)$ ) of subsets of $X_{n-1}$ of cardinality $r-1$ such that $\left|B_{i} \cap B_{i+1}\right|=r-2$ for $i=1, \ldots, r-1$, and

$$
B_{1}=\{n-r+1, \ldots, n-1\}, B_{2}=\{n-r, \ldots, n-2\}, B_{t}=\{1, n-r+2, \ldots, n-1\} .
$$

Let $B_{i}^{\prime}=B_{i} \cup\{n\}$. Then

$$
A_{1}, \ldots, A_{m}, B_{1}^{\prime}, \ldots, B_{t}^{\prime}
$$

is a complete list of the subsets of $X_{n}$ of cardinality $r$. (Notice that $t+m=\left(\begin{array}{l}n \\ r\end{array}\right)$.) Now, arrange the above subsets as follows:

$$
B_{1}^{\prime}, A_{1}, A_{m}, \ldots, A_{2}, B_{2}^{\prime}, \ldots, B_{t}^{\prime} .
$$

Then it is easy to verify that this arrangement satisfies $\mathbf{P}(n, r)$. Hence the induction is complete and we may deduce that $\mathbf{P}(n, r)$ is true for all $n \geq 4$ and all $r$ such that $2 \leq r \leq n-2$. 
ACKNOWLEdgement. My sincere thanks are due to my research supervisor, Professor J. M. Howie, for his helpful suggestions and encouragement.

\section{REFERENCES}

1. G. U. Garba, Nilpotents in semigroups of partial one-one order-preserving mappings, Semigroup Forum, to appear.

2. G. M. S. Gomes and J. M. Howie, Nilpotents in finite symmetric inverse semigroups, Proc. Edinburgh Math. Soc. (2) 30 (1987), 383-395.

3. G. M. S. Gomes and J. M. Howie, On the ranks of certain finite semigroups of transformations, Math. Proc. Cambridge Philos. Soc. 101 (1987), 395-403.

4. G. M. S. Gomes and J. M. Howie, On the ranks of certain semigroups of order-preserving transformations, Semigroup Forum 45 (1992), 272-282.

5. J. M. Howie, An introduction to semigroup theory (Academic Press, 1976).

6. J. M. Howie and R. B. McFadden, Idempotent rank in finite full transformation semigroups, Proc. Roy. Soc. Edinburgh Sect. A 114 (1990), 161-167.

7. R. P. Sullivan, Semigroups generated by nilpotent transformations, J. Algebra 110 (1987), 324-345.

Department of Mathematical and Computational Sciences

UNIVERSITY OF ST ANDREWS

SCOTLAND

Present address:

Department of Mathematics

Ahmadu Bello University Zaria

Nigeria 